\title{
Biomimetic optical sensor for real-time aircraft wing deflection measurement
}

\author{
Susan A. Frost ${ }^{1}$ \\ NASA Ames Research Center, Moffett Field, CA, 94035 \\ Cameron H.G. Wright ${ }^{2}$ and $\mathrm{Md}$. Arif $\mathrm{Khan}^{3}$ \\ University of Wyoming, Laramie, WY 82071
}

\begin{abstract}
Reducing the environmental impact of aviation is a primary goal of NASA aeronautics research. One approach to achieve this goal is to build lighter weight aircraft, which presents complex challenges due to a corresponding increase in structural flexibility. Wing flexibility can adversely affect aircraft performance from the perspective of aerodynamic efficiency and safety. Knowledge of the wing position during flight can aid active control methods designed to mitigate problems due to increased wing flexibility. Current approaches to measuring wing deflection, including strain measurement devices, accelerometers, or GPS solutions, and new technologies such as fiber optic strain sensors, have limitations for their practical application to flexible aircraft control. Hence, it was proposed to use a biomimetic optical sensor based on the common housefly's vision system to track wing deflection in real-time. This fly eye sensor has several advantages over conventional sensors used for this application, including light weight, low power requirements, fast computation, and a small form factor. This paper reports on the fly eye sensor development and its application to real-time wing deflection measurement.
\end{abstract}

\section{Introduction}

$\mathrm{I}_{\mathrm{c}}^{\mathrm{n}}$ an effort to reduce the environmental impact of aviation, lighter weight aircraft configurations are being considered [1]. One challenge of light weight aircraft wings is increased flexibility that can adversely affect handling qualities and safety. Approaches using active control to mitigate problems associated with flexible wings have been proposed [2]-[6]. Knowledge of aircraft wing position during flight can provide significant advantages to the effectiveness of these approaches. Current approaches to measuring wing deflection, including strain measurement devices, accelerometers, or GPS solutions, and new technologies such as fiber optic strain sensors, have limitations for their practical application to flexible aircraft control. For instance, strain gauge measurement systems are quite heavy and need frequent recalibration, reducing their suitability for use in wing position sensing. While fiber optic based strain sensors are showing promise, some issues related to calibrating measured strain with wing twist remain. Traditional machine vision systems using charge coupled device (CCD) or complementary metal oxide semiconductor (CMOS) arrays have several disadvantages for applications requiring high sensitivity to motion and high speed extraction of certain image features such as the object edges of a target, including the blurring of objects moving at high speed, and the high computation and data throughput requirements for edge detection [7]-[8]. A machine vision system that can perform high speed target tracking in near real-time with low power requirements is desirable for wing deflection tracking.

An optical sensor based on the physiological aspects of the eye (and vision-related neural layers) of the common housefly (Musca domestica) has been developed by researchers at the University of Wyoming [9]-[16] and NASA Ames Research Center. A biomimetic (also known as biologically inspired) engineering approach was used to extract salient features of the fly's visual system for use in the optical sensor. The intent of the research is not to completely reproduce the ability, appearance, or function of the fly's vision system. Rather, select features that are desirable in image processing are reproduced, including high functionality in low-light and low-contrast environments, sensitivity to motion, compact size, and low power and computation requirements.

\footnotetext{
${ }^{1}$ Research Scientist, Intelligent Systems Division, M/S 269-3, AIAA Senior Member.

${ }^{2}$ Associate Professor, Dept. of Electrical and Computer Engineering, 1000 E. University Ave.

${ }^{3}$ Student, Dept. of Electrical and Computer Engineering, 1000 E. University Ave.
}

American Institute of Aeronautics and Astronautics 


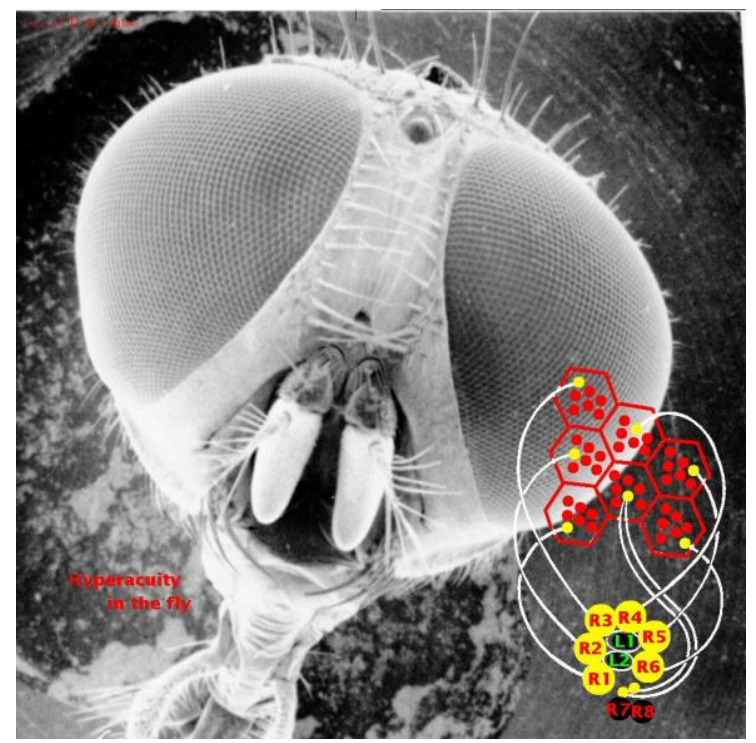

Fig. 1. Scanning electron microscope image of Musca domestica with depiction of associated first neural layer neural superimposed showing neural superposition. Image courtesy of M. Wilcox.

There is a large body of research on the fly's visual system (see fig. 1) that was leveraged to develop the fly eye sensor described here [16]-[20]. Laboratory tests were performed on flies at the University of Wyoming to characterize the arrangement and interaction of light sensitive cells (ommatidia) in the fly's compound eye [16]. The fly uses a combination of quasi-Gaussian overlapping photoreceptor responses and neural superposition to achieve what has been described in the literature as "hyperacuity," or the ability to detect image features, such as object motion, to a much higher degree than just the photoreceptor density would imply. See fig. 2 for an image depicting the overlapping Gaussian response similar to that exhibited by the fly's vision sysem. The overlapping Gaussian response fields-ofview (FoVs) allow for very precise and very fine measurements of position, direction, and speed.

Figure 3 shows a simplified diagram to illustrate the Gaussian overlapping photoreceptor response and neural superposition that both contribute to such desirable sensor capabilities. In this figure, the seven shaded photoreceptors from seven physically distributed ommatidia shown in (a) all view the same point at infinity. The overlapping visual field of the three receptors depicted in (b) is shown as (c). All seven visual fields (as half power radii) are shown perpendicular to the optical axis in (d). Signals from the physically distributed but optically coaligned photoreceptors combine in the neural layer and together are called a "cartridge." While the fly has eight photoreceptors in each ommatidium, receptors R7 and R8 are "stacked" and share a single optical axis. Note that the Gaussian overlap for the artificial sensor comes from the front-end optical design, and the neural superposition is accomplished by how the signals are subsequently combined.

Since the sensor excels in detection of even minute motion, a feasibility study using the sensor for detailed target tracking was proposed. The purpose is to track a known target pattern at relatively short range, and resolve the position
Fig. 2. Depiction of overlapping Gaussian response similar to that exhibited by compound eye of common house fly.

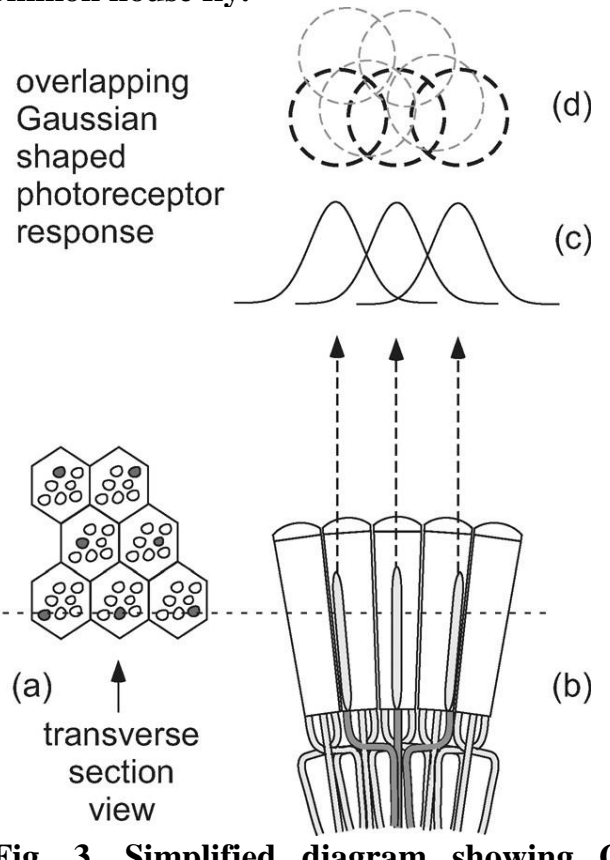

Fig. 3. Simplified diagram showing Gaussian overlapping photoreceptor response and neural superposition of fly eye visual system; each hexagonal shape in (a) delineates the cross section of structure called an ommatidium. 
and velocity of the pattern relative to a neutral position. One application is the precise measurement of wing deflection in a fixed wing aircraft. This optical approach allows for a faster, more efficient approach than alternative methods (such as accelerometers or strain gages within the target object). This method does have drawbacks however, such as its reliance on a clean line-of-sight to the target.

\section{Sensor design and construction}

\section{A. Sensor subsystems}

This section describes the function and composition of each subsystem within the fly eye sensor as designed by Dean [12], which includes lessons learned from several years of development by other researchers [9]-[16]. See fig. 4 for a block diagram of the functionality of one fiber optic channel of the sensor from input to output. The version of the sensor described here uses seven fiber-optically fed IFD91 photodarlington detectors [21]. These semiconductive devices output a current proportional to the number of photons impinging on the element (i.e. the quantity of light in front of the element). This current is small (on the order of $0.1 \mu \mathrm{A}$ to $10 \mathrm{~mA}$ ) and thus requires both conversion to a voltage as well as amplification. Following the photodarlingtons, a logarithmic compression amplifier circuit is used to achieve current-to-voltage conversion and to enable increased dynamic range of the output values. Use of the sensor in a wide variety of ambient lighting conditions, such as from dim light to extremely bright conditions, requires such increased dynamic range to ensure adequate image contrast for detection purposes.

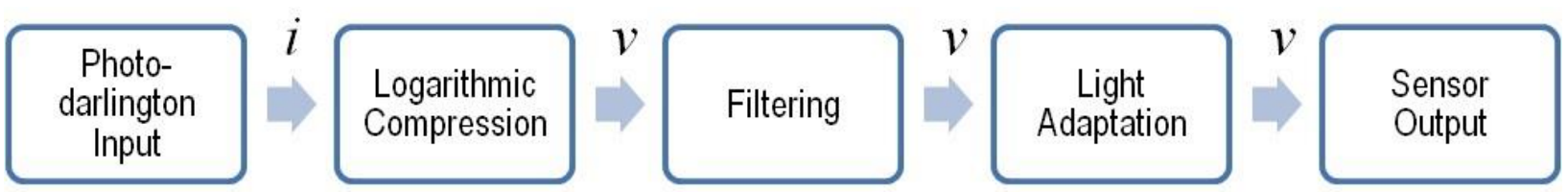

Fig. 4. Block diagram of sensor functionality through a single channel, where $i$ represents current and $v$ represents voltage.

A set of active filters are used to remove noise present in the signal. These filters include a 4th-order Butterworth low-pass filter with a $50 \mathrm{~Hz}$ cutoff frequency and a notch filter centered at $60 \mathrm{~Hz}$. These filters specifically target noise sources that manifest themselves as flicker in interior lighting, which is well within the detection range of the sensor. This is an inexpensive, efficient filtering approach that requires only a small number of components and very little space on the PCB. Since the signal of interest is in the near-DC range, a low-pass filter is used.

Next the signals enter a light adaptation stage that is designed to allow the sensor to operate in a variety of ambient lighting conditions. The light adaptation stage makes use of the average value of the ambient lighting. The signal average is computed using a single-stage averaging amplifier arrangement. This circuit is a variation on a noninverting summing amplifier circuit, in which the gain for the signals coming from all 7 optical axes is set to approximately 1/7. The computed average is then filtered to near DC using a single stage active filter. This filter reduces oscillations caused by detected motion in the computation of the average, and thus mitigates the carry-over of those oscillations into the sensor output signals. The filter is only applied to the computed average value, and is not present in all 7 signal channels. The average is designed to accommodate all seven channel voltages. If a particular application requires fewer sensor channels (such as mono-axial tracking), and the sensor needs to be manufactured as inexpensively as possible, this circuit can be redesigned.

Following computation of the average value, an instrumentation amplifier is used to subtract the average from each of the 7 signals independently. This operation achieves what is referred to as "light-adaptation" through mean removal, a process by which the sensor can adapt to different levels of ambient lighting [12]. A low-power instrumentation amplifier is used to perform this subtraction. The outputs from this stage are considered the final sensor output signals, which can then be sampled by a computer.

Used by itself, the light adaptation circuit reacts almost instantaneously, which is detrimental to the sensor performance. Light adaptation is meant to adjust for relatively slow changes in ambient lighting to allow a wider dynamic range of operation than would otherwise be possible. It was found, not unexpectedly, that if the light adaptation is allowed to be too fast, it will "adapt" to light changes due to objects of interest instead of to ambient lighting, and make objects of interest harder to detect. The solution is to add a time delay to the light adaptation subsystem. All biological creatures incorporate such a time delay into their light adaptation physiology.

Traditional electronic time delay methods, such as memory/shift registers, are not feasible due to the need to preserve the analog nature of the signal. A method employing analog-to-digital conversion followed by a delay

American Institute of Aeronautics and Astronautics 
followed by digital-to-analog conversion would be too expensive in terms of computation power and time and it could introduce too much quantization error. Older analog methods such as resistive-capacitive (RC) or resistive-inductive (RL) delay lines suffer from poor stability and unrealistic component values for the delay needed, which was determined to be approximately one second. The resulting solution includes a sample and hold circuit with a timer that samples the analog signal, but does not quantize or digitally encode the signal. Thus delay was achieved without compromising the analog nature of the sensor signal [12].

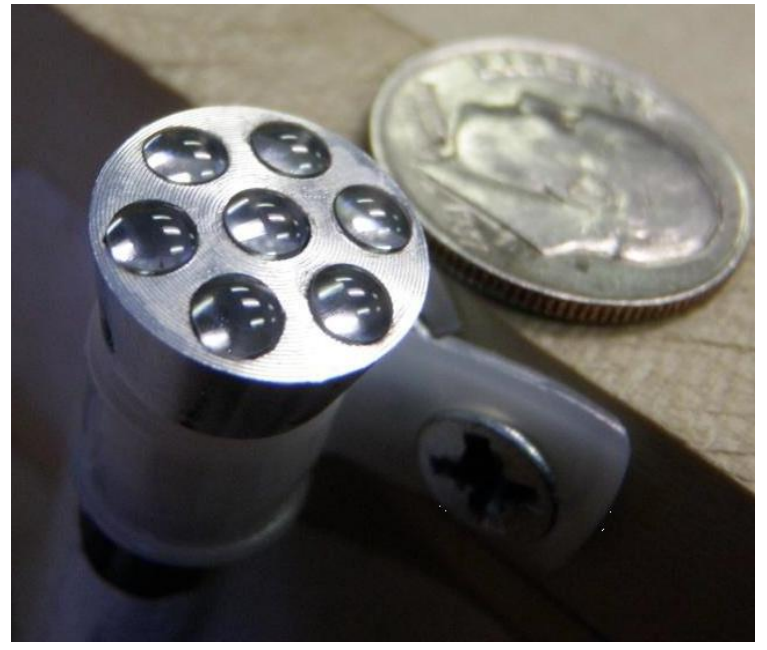

Fig. 5. Front view of sensor head with U.S. dime for comparison.

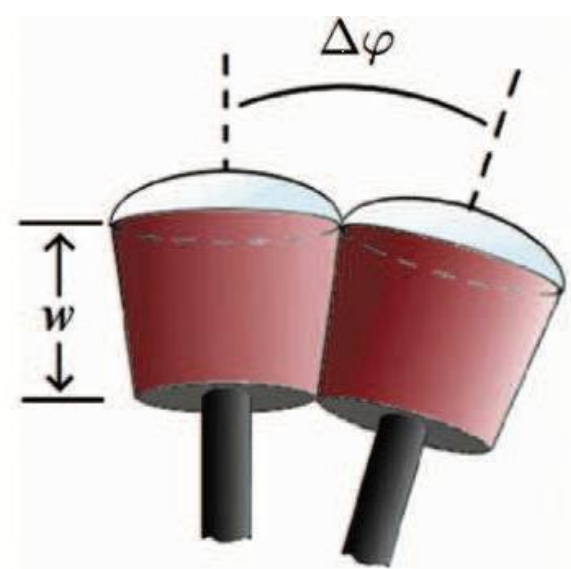

Fig. 6. Detail of two photo receptors in sensor head, where $w$ represents the distance between the lens and the image plane and $\Delta \varphi$ represents the angle between two lenses.

Once the sensor is physically assembled, it must be properly calibrated within the desired test environment. The sensor is calibrated by directing all facets toward an evenly lit, single-color, specular reflection free background and then adjusting the $50 \mathrm{k} \Omega$ potentiometers so that all outputs (one from each of the seven channels) are as near to the same value as possible. In an ideal lab setup, a curved Lambertian surface would be used to ensure that each of the seven channels is receiving stimulus that is not dependent on the viewing angle. This arrangement cannot be achieved with the facilities available, so an alternative approach is used. The maximum output voltage difference measured after calibration in the lab is approximately 0.682 volts (about $5.4 \%$ of full-scale). An automatic calibration technique using digital potentiometers can also be used [12].

A hyperacuity to motion is a compelling attribute of this sensor package, and is one of the main reasons for this research. As a target moves within the FOV of the sensor, the response is nearly immediate. The photodarlingtons

American Institute of Aeronautics and Astronautics 
measure the quantity of light within their range. For example, a target that is darker than the background causes a decrease in the output of a photodarlington sensing the target. Tracking multiple outputs at once allows for the determination of the direction and speed of such a target.

Table 1. Characterization parameters for sensor measured over numerous trails.

\begin{tabular}{|l|l|l|l|}
\hline Parameter & Min & Typical & Max \\
\hline Output [V] & $\sim 0.0$ & 0.49 & 0.72 \\
\hline Operating Range [cm] & & 25.4 & $\sim 130-150$ \\
\hline Sensor, PCB, and Cables Weight [g] & & 135 & \\
\hline Input Illuminance [lx] & 1 & 520 & 36,000 \\
\hline Power Consumption [W] & & 3.3 & \\
\hline
\end{tabular}

Characterization tests have indicated a number of operating parameters for the system, see Table 1 . These describe general operating conditions for the sensor package. Extreme conditions may result in different behavior. The logarithmic compression system is designed to operate up to 72,000 luminance (or lux) [12], which, as Table 1 indicates, is far above the highest generated in the lab setting. While the operating range between the sensor and the target indicated in Table 1 is sufficient for the demands of this project, augmented range may be realized for other applications by using additional optics in front of the sensor head.

\section{Sensor Simulation}

A MATLAB ${ }^{\mathrm{TM}}$ simulation program has been developed for the fly eye sensor described in the previous section. This program can simulate the sensor output for a moving target of arbitrary shape and size against a background. The target-background contrast can be varied, and the target movement can be controlled in front of the sensor. The simulation has the flexibility of enabling a change in the sensor to target distance and the relative orientation of the sensor and target. The assumption of paraxial theory of light together with small angle approximation is used to simulate the sensor response to the target-background combination. This assumption is found valid from the simulation results, because the simulation program incorporates only the total amount of light from the scene that is brought to focus on the tip of the fibers. Uniformity of the light intensity, and Lambertian reflectance throughout the surface of the background is also assumed. The output of the sensor is deemed ideal (disregarding the non-ideality due to higher order optical properties such as diffraction of the lenses). A correction factor can be introduced to match the simulated output to the actual sensor output. Also, noise with different distributions and power levels can be added to the

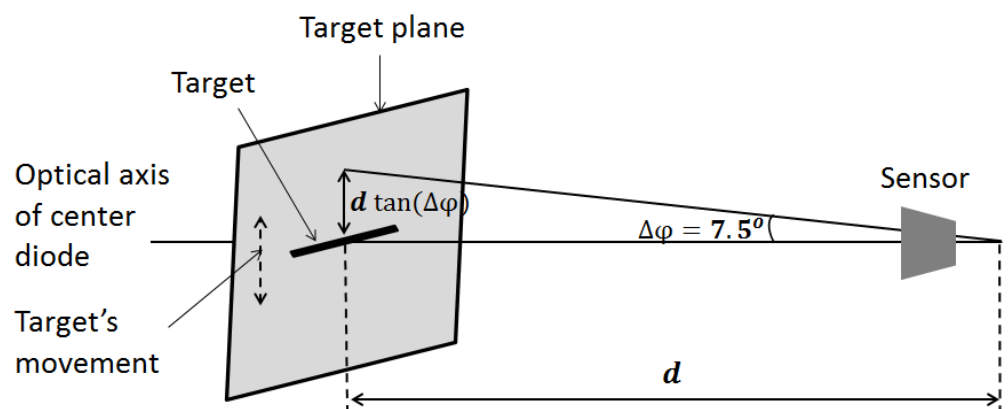

(a)

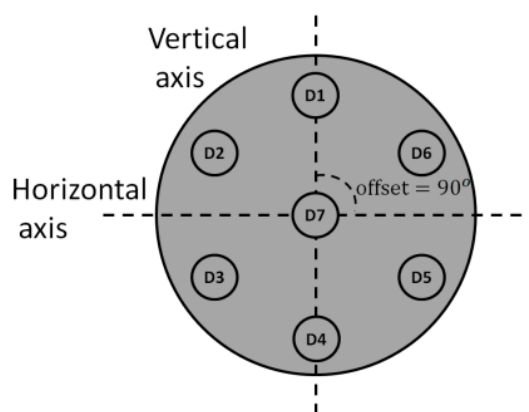

(b)

Fig. 7. (a) Simulation experimental setup. (b) Sensor-target relative orientation

simulated output to analyze the real world application scenario.

Figure 7a shows the experimental setup used for simulating a moving target on the target plane at a certain distance $\mathrm{d}$ from the virtual converging point of the sensor housing. The inter-lens angle $\Delta \varphi=7.5 \mathrm{~d}$ comes from the optical front end design, which increases motion sensitivity at small object distances [2]. At the same time, an overlap of 50\% between adjacent pixel responses has been used to maximize the motion detection capability [2]. Since, the sensor is 
designed to have $50 \%$ overlap at all object distances (but not beyond the optical infinity of the sensor, which is about $1 \mathrm{~m}$ [13]), the angular roll off of an individual lens' response must decrease with the increase in object distance. This implies the spread of the Gaussian response must increase with the increase in sensor-target distance (as shown in Figure 7 for inter-lens angle $0 \mathrm{~d}$ instead of $7.5 \mathrm{~d}$ ). The spread $\sigma$ that provides $50 \%$ overlap between adjacent pixels over all usable object distances (i.e., object distance less than $1 \mathrm{~m}$ ) is a function of distance $d$ and inter-lens angle $\Delta \varphi$ (as shown in Figure 7), and expressed as

$$
\sigma=\frac{d \tan \left(\frac{\Delta \varphi}{2}\right)}{\sqrt{\ln (4)}}
$$

This is drawn from two 50\% overlapping standard Gaussian equations with the spread $\sigma$ for both.

The sensor orientation relative to the target's movement direction is described with an additional parameter "offset", which is simply the angle between the horizontal axis, and the position of the optical element interfaced with the photo-diode D1 of channel 1 (the first pixel). The parameter "offset" is introduced here to describe how much the sensor head has to be rotated for a particular application with respect to the horizontal axis (i.e., the axis parallel to the ground). Figure $7 \mathrm{~b}$ shows the sensor orientation with offset $=90 \mathrm{~d}$, where D1 to D7 denote the positions of the seven photodiodes associated with the seven optical axes of the sensor. This arrangement is done to make the target movement vector (up-down) parallel to the line passing through diodes D1, D7, D4.

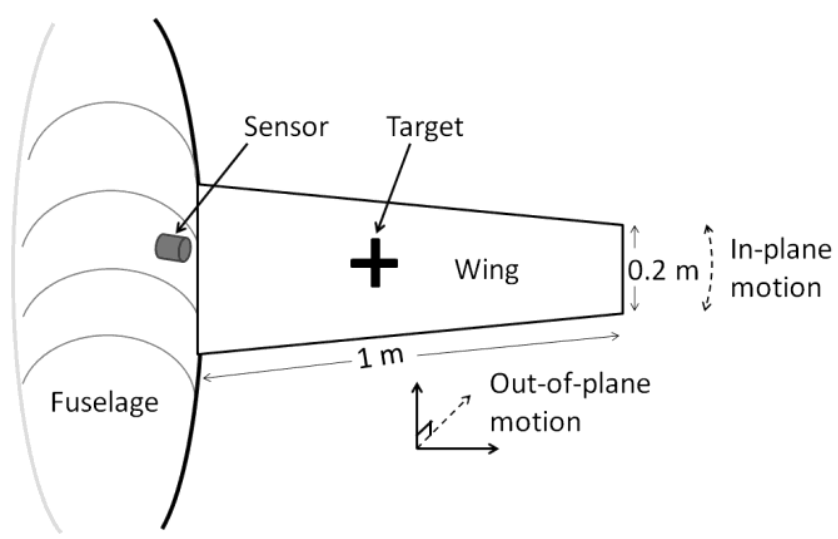

Fig. 8. Sensor-target arrangement on an aircraft.

\section{Application Specification}

A key requirement of the application proposed here is to measure the deflection of an aircraft wing from its resting position in real or near-real time. We consider a small Unmanned Aerial System (UAS), where the wing can bend a maximum of $3 \mathrm{~cm}$ from its resting position, with oscillation frequencies of less than $3 \mathrm{~Hz}$. The sensor-target distance would be about $1 \mathrm{~m}$, and the size of the target can be increased up to $25 \mathrm{~cm}$ in width. The height of the target can be increased up to the span of a wing (which is very large compared to the FOV of the sensor for this application). That is, we have some flexibility in determining the shape and size of the target, and the distance between the sensor and the target. The wing deflection may include bending and twist, but for this research twist is ignored. So, the key goal of this research is the real, or near-real, time tracking of a linearly moving target. A top level diagram of the sensor-target arrangement is shown in Figure 8, where the sensor is mounted on the fuselage of the aircraft, and the target is painted on the surface of the wing. The sensor is looking down at the wing and out the wing. The out-of-plane bending motion of the wing is defined as when the wing bends in the direction up or down. Measuring the torsion or twist of the wing is a long term goal of the project, but for now, the most important measure is the out-of-plane bending measured by one target on the wing.

Many researchers report on studies to estimate out-of-plane displacements by measuring in-plane strains. Using a cantilevered plate in the laboratory, Haugse et al. [22, 23] developed a modal transformation algorithm to recover deformations from strains. In a similar procedure, Pisoni and Santolini [24] determined the displacements at any given point in a vibrating clamped-end beam under different loading conditions using two strain gages. Li and Ulsoy [25] presented a method to measure tool-tip displacement of precision line boring machine. Davis et al. [26] and Kim et al. [27] measured vertical deflections of a simple beam models using fiber Bragg grating (FBG) sensor signals. The key concept of these strain based techniques is the vibration displacement can be expressed in terms of an infinite number of vibration modes and be related to the measured strains through the displacement-relationship. Most of the developed techniques used conventional strain gages, which need complex wiring in order to measure the strains at several points. Also, most of them can only detect static deformations or the deflections at few interesting points of the moving structures. These indirect shape estimation techniques using strain measurements, which can come from foil gages and/or fiber-optics Bragg gratings (FBG) become inefficient because of the weight of the gages, and cost associated

American Institute of Aeronautics and Astronautics 
with strain-displacement transformation. Besides these, airplane may go under extreme temperature changes which greatly affects the sensitivity of the strain

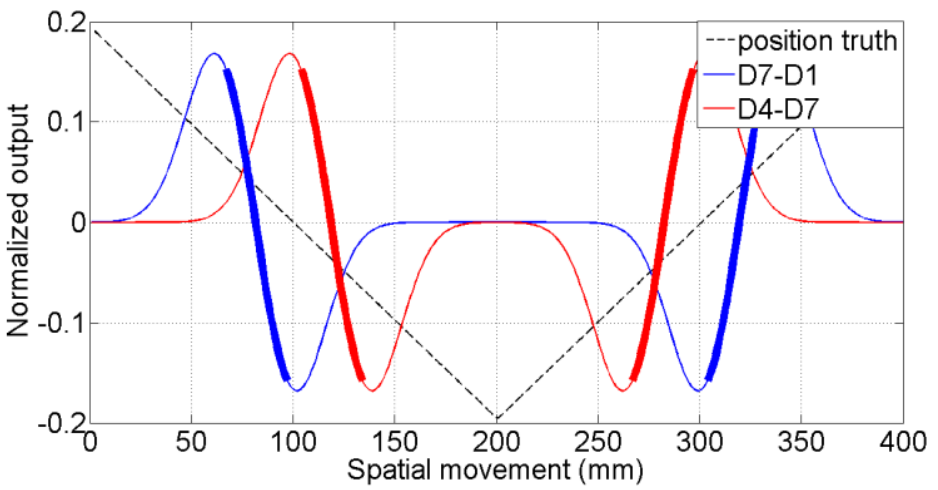

Fig. 9. Inter-pixel differences of the sensor output for a moving target placed at $\mathrm{d}=\mathbf{2 8} \mathrm{cm}$ with offset $=90$.

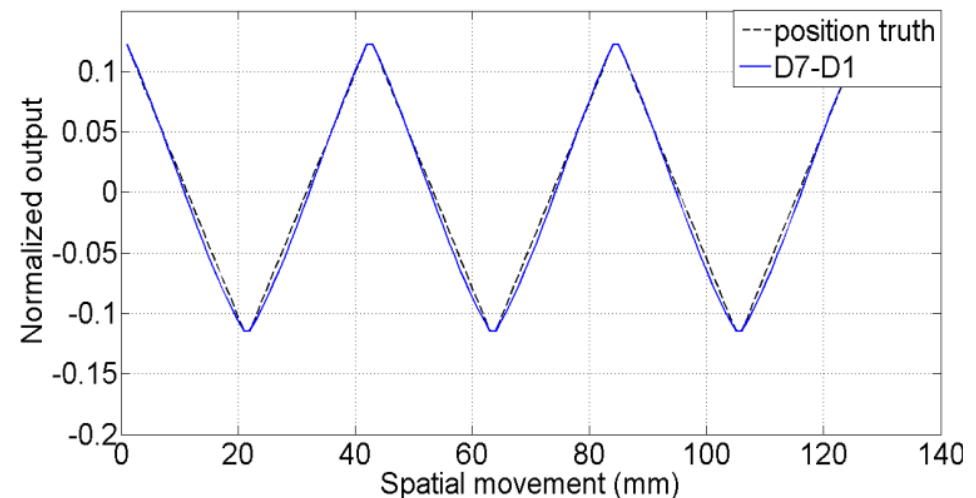

Fig. 10. Real-time tracking of a target which movement was limited within the region shown as blue thick line in Figure 9.

gages, and also requires external excitation source for their operations.

\section{A.Simulation Study}

The application scenario described in the previous section was analyzed using the simulation program. A black target of size 7 $\mathrm{mm} \times 511 \mathrm{~mm}$ was placed at a distance $d=$ $28 \mathrm{~cm}$ (the definition of $d$ was shown in Figure 7a) from the sensor, and swept twice vertically (i.e., offset $=90 \mathrm{~d}$ ) within the FOV of the sensor and beyond that. The distance $d=28 \mathrm{~cm}$ was chosen to have a sensor-target distance of about $25 \mathrm{~cm}$ for the actual setup. The sensor output was simulated for this setup, and the inter-pixel differences were calculated. The results are shown in Figure 9.

It is interesting to note the quasi-linearity of the inter-pixel difference results over certain displacements of the target (shown as thicker lines in Figure 9). This implies that direct mapping of the inter-pixel differences to a deflection measurement without any sophisticated algorithm is viable, if we can somehow restrict the movement of the target within this quasi-linear region. Therefore, the displacement of the target was limited to one of these regions (the region under the blue thicker lines in Figure 9, which is about 3.2 $\mathrm{cm})$. The sensor output was simulated under these conditions, and then the inter-pixel difference D7-D1 was calculated.

The result of the tracking algorithm for this test condition is shown in Figure 10. Note that the "position truth" of this figure is scaled to match the result for comparison purposes. From the figure, it is obvious that the inter-pixel difference is congruent with the position of the target (disregarding a small nonlinearity in the result). Thus, the inter-pixel difference alone can be used to determine the instantaneous position of a moving target, when the target movement is limited as described above. This result seems promising, and therefore, it was implemented in a circuit using the actual sensor hardware. The details of the implementation and results using the actual sensor are described in the following section.

\section{B. Sensor Hardware Study}

A study was performed to track a simple target moving in a plane in front of the sensor. The distance between the top surface, and the virtual converging point of the actual sensor head housing was measured, and found to be approximately $3 \mathrm{~cm}$. A vertical black target of width around $7 \mathrm{~mm}$ was placed at distance $25 \mathrm{~cm}$ from the top surface of the sensor head, resulting in a sensor-target distance of about $d=28 \mathrm{~cm}$. The target was moved horizontally in front of the sensor, with its movement limited to around $3.8 \mathrm{~cm}$. The outputs of all seven channels were measured before the light adaptation stage of the signal conditioning and processing hardware. This was done to avoid complications

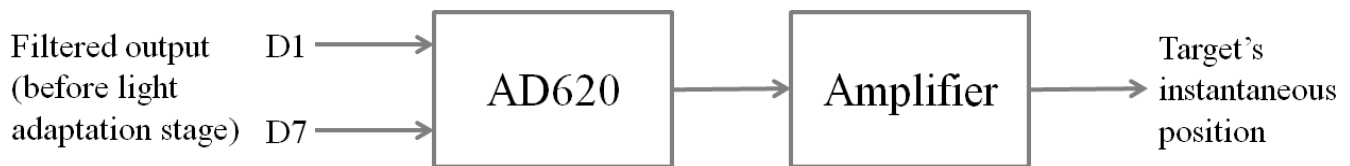

Fig. 11. Circuit for processing sensor output to perform real-time tracking of a moving target.

American Institute of Aeronautics and Astronautics 
due to the near DC filter, and the average subtraction process of the light adaptation stage. The inter-pixel difference was determined using an instrumentation amplifier from Analog Devices (an AD620). The system is extremely simple, see Figure 11. The amplification stage, as shown in Figure 11, was used to scale the system output. This stage can be replaced with an interfacing circuit based on the requirements of the system that can be cascaded for further manipulation of the tracking result.

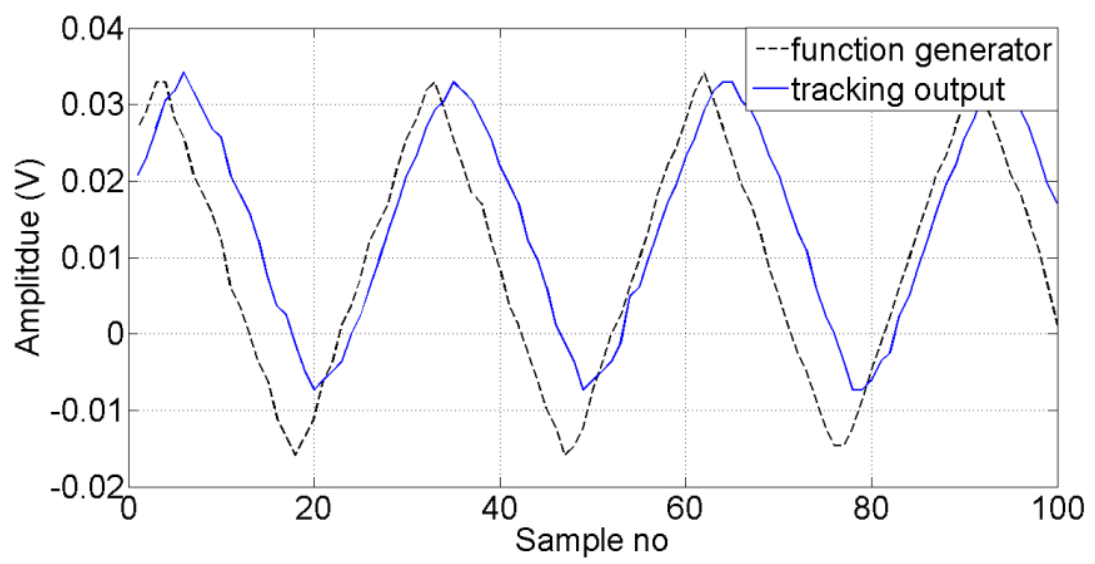

Fig. 12. Real-time tracking of a moving target using a fly eye sensor. The time delay, amplitude, and the bias in the tracking output can be adjusted as desired. Note, the signals are plotted against sample number instead of the spatial movement to show that results are not dependent on the sampling rate.

The output of the tracking system is analog, and was observed using an oscilloscope. The output was sampled with a $30 \mathrm{~Hz}$ sampling frequency. The sampled data is shown in Figure 12 (blue solid line) along with the signal from the function generator that drives the target (black dashed line). Note, the system output shown in this figure is just a raw signal from the circuit without any kind of processing (the amplifier gain was set to 1 ). The time delay between the signal from the function generator and the system output is expected due to the mechanical delay of the plotter, imprecise positioning of the target in front of the sensor, and the delay from the signal conditioning and processing circuit. The bias in the output is due to the signal acquisition process using a DATAQ DI148U. Disregarding the amplitude level, the output clearly shows the real-time tracking capability of the system.

One important point to be observed in the system output is a small amount of noise. The noise is expected due to the test setup. During the test, a vertical target with an unsupported top end was used. The unsupported end of the target vibrates when the target moves horizontally. Therefore, the noise in the output is actually capturing the instantaneous position of the vibrating target in front of the sensor.

The output of the tracking system was observed when placing the target at different distances from the sensor, and in different ambient lighting conditions. From the results, it was found that the tracking system can successfully track the moving target, but the amplitude of the tracking system output varies with the variation in ambient light intensity, and sensor-target distance.

\section{Conclusions}

This paper reports on efforts to develop a biomimetic fly eye sensor to track a target for real-time measurement of aircraft wing deflection. The fly eye sensor has several advantages over conventional sensors used for this application, including light weight, low power requirements, fast computation, and a small form factor. Tests were run in which data was obtained from the sensor when a target moved in a horizontal direction in front of the sensor, simulating an aircraft wing moving under bending loads. A simple differencing algorithm that was implemented in hardware showed good target tracking results providing a real-time solution to tracking the deflection of an aircraft wing.

\section{Acknowledgments}

This work was performed with support from the NASA Aeronautics Research Institute.

\section{References}

[1] NASA Aeronautics Research Mission Directorate, [Online]. Available: http://www.aeronautics.nasa.gov/, Jan. 27, 2014.

[2] L. Librescu, and P. Marzocca, "Advances in the linear/nonlinear control of aeroelastic structural systems," Acta Mechanica 178.3-4 (2005): 147-186.

American Institute of Aeronautics and Astronautics 
[3] M.J. Brenner, R.C. Lind, and D.F. Voracek. Overview of recent flight flutter testing research at NASA Dryden. Vol. 4792. National Aeronautics and Space Administration, Office of Management, Scientific and Technical Information Program, 1997.

[4] A. Derkevorkian, S. F. Masri, J. Alvarenga, H. Boussalis, J. Bakalyar, and W. L. Richards, "Strain-Based Deformation Shape-Estimation Algorithm for Control and Monitoring Applications", AIAA Journal, Vol. 51, No. 9 (2013), pp. 2231-2240, doi: 10.2514/1.J052215.

[5] P.M. Suh and D.N. Mavris, "Modal filtering for control of flexible aircraft", Proceedings $54^{\text {th }}$ AIAA/ASME Structures, Structural Dynamics, and Materials Conference, Boston, MA, April 2013.

[6] P.M. Suh, A.W. Chin, and D.N. Mavris, "Virtual Deformation Control of the X-56A Model with Simulated Fiber Optic Sensors," Proceedings AIAA Atmospheric, Flight Mechanics Conference, Boston, MA, Aug. 2013.

[7] G. C. Holst, Electro-Optical Imaging System Performance, 3rd ed. SPIE Press, 2003.

[8] R. H. Vollmerhausen, D. A. Reago, Jr., and R. G. Driggers, Analysis and Evaluation of Sampled Imaging Systems, SPIE Press, 2010.

[9] J. D. Davis, S. F. Barrett, and C. H. Wright, "Bio-inspired minimal machine multi-aperture apposition vision system," ISA Biomedical Sciences Instrumentation, vol. 44, 2008, pp. 373-379.

[10] D. T. Riley, W. Harman, S. F. Barrett, and C. H. G. Wright, "Musca domestica inspired machine vision sensor with hyperacuity," IOP Journal of Bioinspiration \& Biomimetics, vol. 3, no. 2, Jun. 2008.

[11] J. B. Benson, C. H. G. Wright, and S. F. Barrett, "Redesign and Construction of an Artificial Compound Eye Visual Sensor," ISA Biomedical Sciences Instrumentation, vol. 44, pp. 367-372, Apr. 2008.

[12] B. Dean, "Light adaptation and applications for a fly eye vision sensor," Ph.D. dissertation, University of Wyoming, 2012.

[13] G. P. Luke, C. H. G. Wright, and S. F. Barrett, "A multiaperture bioinspired sensor with hyperacuity," IEEE Sensors Journal, vol. 12, no. 2, pp. 308-314, February 2012.

[14] R.W. Streeter, "Target tracking with a Musca domestica based sensor platform," M.S. thesis, University of Wyoming, 2013.

[15] R. S. Prabhakara, C. H. G. Wright, and S. F. Barrett, "Motion detection: A biomimetic vision sensor versus a ccd camera sensor," IEEE Sensors Journal, vol. 12, no. 2, pp. 298-307, February 2012.

[16] E. Tomberlin, "Musca Domestica's large monopolar cell responses to visual stimuli," Master's thesis, University of Wyoming, June 2004.

[17] K. Nakayama, "Biological image motion processing: A review," Vision Res., vol. 25, pp. 625-660, 1985.

[18] J. S. Sanders and C. E. Halford, "Design and analysis of apposition compound eye optical sensors," SPIE Optical Engineering, vol. 34, no. 1, pp. 222-235, Jan. 1995.

[19] M. F. Land and D. Nilsson, Animal Eyes. Oxford, U.K.: Oxford Univ. Press, 2002.

[20] B. G. Burton and S. B. Laughlin, "Neural images of pursuit targets in the photoreceptor arrays of male and female houseflies Musca domestica," J. Exp. Bio., vol. 206, pp. 3963-3977, 2003.

[21] Plastic Fiber Optic Photodarlington, Industrial Fiber Optics, Inc., 2006. [Online]. Available:http://www.fiberoptics.com/

[22] P. B. Bogert, E. Haugse, and R. E. Gehrki, "Structural shape identification from experimental strains using a modal transformation technique," Proceedings 44th AIAA/AS-ME/ASCE/AHS/ASC Structures, Structural Dynamics, and Materials Conference, 2003.

[23] G. C. Foss and E. D. Haugse, "Using modal test results to develop strain to displacement transformation," IMAC, vol. 1, pp. 112-118, 1995.

[24] A. Pisoni and C. Santolini, "Displacements in vibrating body by strain gauge measurements," IMAC, vol. 1, pp. $119-125,1995$.

[25] C. J. Li and A. G. Ulsoy, "High-precision measurement of tool-tip displacement using strain gauges in precision flexible line boring," Mechanical Systems and Signal Processing, vol. 13, pp. 531-546, 1999.

[26] P. B. Bogert, E. Haugse, and R. E. Gehrki, "Structural shape identification from experimental strains using a modal transformation technique," Proceedings 44th AIAA/ASME/ASCE/AHS/ASC Structures, Structural Dynamics, and Materials Conference, 2003.

[27] C. J. Li and A. G. Ulsoy, "High-precision measurement of tool-tip displacement using strain gauges in precision flexible line boring," Mechanical Systems and Signal Processing, vol. 13, pp. 531-546, 1999.

American Institute of Aeronautics and Astronautics 